\title{
Coordinate space representation for renormalization of quantum electrodynamics
}

\author{
Amirhosein Mojavezi,, * Reza Moazzemi, ${ }^{2, \dagger}$ and Mohammad Ebrahim Zomorrodian ${ }^{1}$ \\ ${ }^{1}$ Department of Physics, Ferdowsi University of Mashhad, 91775-1436 Mashhad, I.R. Iran \\ ${ }^{2}$ Department of Physics, University of Qom, Ghadir Blvd., Qom 371614-6611, I.R. Iran
}

\begin{abstract}
In this paper we present a systematic treatment for fundamental renormalization of quantum electrodynamics in real space. Although the standard renormalization is an old school problem in this case, it has not yet been completely done in position space. The most important difference with well-known differential renormalization is that we do the whole procedure in coordinate space without need to transformation to momentum space. Specially, we directly derive the conterterms in real space. This problem becomes important when the translational symmetry of the system breaks somehow explicitly (for example by nontrivial boundary condition (BC) on the fields). In this case, one is not able to move to momentum space by a simple Fourier transformation. Therefore, in the context of renormalized perturbation theory, by imposing the renormalization conditions, counterterms in coordinate space will depend directly on the fields BCs (or background topology). Trivial BC or trivial background lead to the usual standard conterterms. If the counterterms modify then the quantum corrections of any physical quantity are different from those in free space where we have the translational invariance. We also show that, up to order $\alpha$, our counterterms are reduced to usual standard terms derived in free space.
\end{abstract}

PACS numbers: 11.10.Gh, 11.15.Bt, 11.10.z, 03.70.+k

\section{INTRODUCTION}

From its early stages, quantum field theory (QFT) was encountered some infinities leading to meaningless results and required to be eliminated. These ultraviolet (UV) infinities are related to the quantum corrections of some physical quantities, such as electron mass and charge [1. Very much attempts, starting with Kramers at 1940s 2], have been done to control and remove these ultraviolet divergences (see for instance [3]). In fact, to calculate a physical quantity (e.g. electron mass) in an interacting field theory, in addition to its 'bare' value, we must take into account quantum corrections, $\Delta m$ :

$$
m_{\text {physical }}=m_{\text {bare }}+\Delta m,
$$

where $\Delta m$ is almost infinite due to undetermined momenta in loop quantum corrections.

Renormalization technique, is a recipe which consistently not only removes but also controls all infinities appeared in the theory (for a qualitative review see for example 4, 5]). The importance of the renormalization procedure is not only to absorb divergences but also to complete the definition of the quantized field theory, i.e., the finite parts of the renormalization constants -fixed by the renormalization conditions- influence the results of the calculation of radiative corrections and therefore of physically observable quantities [6. In QFT, there are two completely equivalent methods for the systematic of renormalization; first, bare perturbation theory: working with the bare parameters and relate them to their physical values at the end of calculations. Roughly speaking, the divergences are absorbed by redefinition of unmeasurable bare quantities (see [7-9]). Second, renormalized perturbation theory: splitting the parameters appeared in the Lagrangian into two parts from beginning: physical part and counterterm that absorbs unphysical part. In fact, the unobservable shifts between the bare and the physical parameters are absorbed by counterterms (see for instance [10 13]). Both methods are required to give us precise definitions of the physical mass and coupling constants by applying renormalization conditions. The differences between two renormalization procedures are purely a matter of bookkeeping $14-16$.

There are many investigations related to renormalization programs concerned with quantum electrodynamics (QED) [17-20], Quantum chromodynamics (QCD) 21-23], and scalar field with various self interactions ([24-27]). All of these theories are renormalizable in four spacetime dimensions, since their coupling constants are dimensionless (Weinberg theorem) 28. On the other hand, renormalization group (RG) methods have been vastly considered too(see for instance 2932 ).

\footnotetext{
*Electronic address: amojavezi98@gmail.com

${ }^{\dagger}$ Electronic address: $r$ r.moazzemi@qom.ac.ir
} 
We should do, in principle, the renormalization in position space. However, for the ease of calculation we do it in momentum space. In fact, there is a duality transformation from $p$ - to $x$-space renormalization specially when we have translational symmetry. One moves from position to momentum space by a simple Fourier transformation. This is easy to do if our wave functions are plain waves. But, if the translational symmetry breaks somehow explicitly, then the momentum is not a good quantum number and the wave functions are not plain waves, so that the transformation to momentum space is no longer so simple and trivial. In this case, field propagators will depend on nontrivial properties that break translational symmetry (e.g. nontrivial boundary conditions (BC) or nontrivial background), therefore, all $n$-point functions and consequently all counterterms will depend on those nontrivial properties. (Please note that, it is not possible to remedy the renormalization in momentum space by any perturbation, since a nontrivial $\mathrm{BC}$ or a nonzero background is not a perturbative phenomenon [33.)

We should here note that Differential Renormalization (DR) procedure [34, 35, which has been vastly investigated in the literature, is done in coordinate space, though the traditional method of renormalization in momentum space (for review see [36, 37]). DR is equivalent to traditional renormalization [38 40], and is based on the observation that the UV divergence reflects in the fact that the higher order amplitude cannot have a Fourier transform into momentum space due to the short-distance singularity. Thus one can, first, regulate such an amplitude by writing its singular parts as the derivatives of the normal functions, which have well defined Fourier transformation, and second, by performing the Fourier transformation in partial integration and discarding the surface term, directly get the renormalized result. In this procedure the surface terms which are dropped during the renormalization are just correspond to the counterterms. Therefore, to get the hidden counterterms we have to move to momentum space again.

The derivation of standard counterterms from scattering amplitudes has been investigated from many years ago 42 45. In the context of DR there also exist some works in massive and massless QED [46, 47]. However, its program in position space has not yet been surveyed. In addition, the large order behavior of $\phi^{4}$ theory for nonzero background field is considered in [48. Also this theory in $1+1$ dimensions, renormalization in real space has been done in Ref. 33. Applications of the theory, where we have nontrivial BCs such as Dirichlet BC or nonzero background such az a kink have been used in Refs. [49] and [52, respectively. In $3+1$ dimensions it has partially done in Ref. [51]. In [53 and 54 perturbative QFT in configuration space has been developed on curved space. Also, one can follow several recent works, for example, amplitudes in a massless QFT [55] and relativistic causality and position space renormalization [56].

In this paper, we shall systematically derive the countetrerms by imposing reasonable renormalization conditions in configuration space where there exist some nontrivial BC. As a matter of fact, the resultant counterterms should be equivalent to ones derived by standard renormalization in momentum space where we have a translational invariance. We will also present this in our paper.

We have organized the paper as the following: We briefly review systematics of renormalization of QED theory in momentum space in Sec. II] Renormalized perturbation theory of QED as a systematic program in position space is considered in Sec. III. In Sec. IV] we compare our results with those in momentum space. Sec. V] summarizes our results and conclusions.

\section{RENORMALIZATION OF QED IN MOMENTUM SPACE: A BRIEF REVIEW}

In this section we briefly review systematics of renormalization for QED theory in momentum space (for complete details see [42]). In general, any renormalizable QFT involves only a few superficially divergent amplitudes. In

QED there are three amplitudes, involving four infinite constants; vertex correction

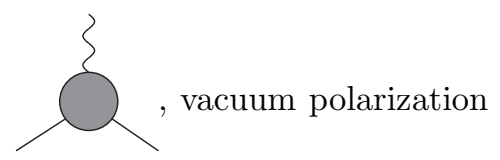

W and electron self energy $\longrightarrow$. The aim of renormalized perturbation theory of QED is to absorb these

constants into the four unobservable parameters of the theory: the bare mass, the bare coupling constant, the electron field strength and the photon field strength. The original QED Lagrangian is

$$
\mathcal{L}_{\mathrm{QED}}=-\frac{1}{4}\left(F^{\mu \nu}\right)^{2}+\bar{\Psi}\left(i \not \partial-m_{0}\right) \Psi-e_{0} \bar{\Psi} \gamma_{\mu} \Psi A^{\mu}
$$


where $m_{0}$ and $e_{0}$ are the bare mass and the bare electric charge, respectively. The $\Psi(x)$ and $A^{\mu}(x)$ are fermion and photon fields, respectively, and can be written as

$$
\begin{aligned}
\Psi(x) & =\int \frac{d^{3} \mathbf{p}}{(2 \pi)^{3}} \sum_{s=1,2} \frac{1}{\sqrt{2 E_{\mathbf{p}}}}\left[c_{\mathbf{p}}^{s} \psi^{s}(x)+d_{\mathbf{p}}^{s \dagger} \phi^{s}(x)\right] \\
A_{\mu}(x) & =\int \frac{d^{3} \mathbf{p}}{(2 \pi)^{3}} \sum_{s=0}^{3} \frac{1}{\sqrt{2 \omega_{\mathbf{p}}}}\left[a_{\mathbf{p}}^{s} \widetilde{A}_{\mu}^{s}(x)+a_{\mathbf{p}}^{s \dagger} \widetilde{A}_{\mu}^{s^{*}}(x)\right],
\end{aligned}
$$

where, in the first line, $c_{\mathbf{p}}^{s \dagger}\left(c_{\mathbf{p}}^{s}\right)$ and $d_{\mathbf{p}}^{s \dagger}\left(d_{\mathbf{p}}^{s}\right)$ create (annihilate) a fermion and anti-fermion with momentum $\mathbf{p}$ and spin direction $s$, respectively. Here, $\psi^{s}(x)$ and $\phi^{s}(x)$ are the particle and anti-particle solutions of the Dirac equation, respectively. In the second line, $a_{\mathbf{p}}^{s \dagger}\left(a_{\mathbf{p}}^{s}\right)$ creates (annihilates) a photon with momentum $\mathbf{p}$ and polarization $\varepsilon_{\mu}^{s}(\mathbf{p})$, and $\widetilde{A}_{\mu}^{r}(x)$ are the momentum-space solution of the equation $\partial^{\mu} A_{\mu}=0$.

By replacing $\Psi(x)=\sqrt{z_{2}} \Psi_{r}(x)$ and $A^{\mu}(x)=\sqrt{z_{3}} A_{r}^{\mu}(x)$, we have

$$
\mathcal{L}_{\mathrm{QED}}=-\frac{1}{4} z_{3}\left(F_{r}^{\mu \nu}\right)^{2}+z_{2} \bar{\Psi}_{r}\left(i \not \partial-m_{0}\right) \Psi_{r}-e_{0} z_{2} \sqrt{z_{3}} \bar{\Psi}_{r} \gamma_{\mu} \Psi_{r} A_{r}^{\mu}
$$

where $z_{2}$ and $z_{3}$ are the field-strength renormalizations for $\Psi$ and $A^{\mu}$, respectively. We define a scaling factor $z_{1}$ as $e z_{1}=e_{0} z_{2} \sqrt{z_{3}}$ and split each term of the Lagrangian into two pieces

$$
\begin{aligned}
\mathcal{L}_{\mathrm{QED}}= & -\frac{1}{4}\left(F_{r}^{\mu \nu}\right)^{2}+\bar{\Psi}_{r}(i \not \partial-m) \Psi_{r}-e \bar{\Psi}_{r} \gamma^{\mu} \Psi_{r} A_{r}^{\mu} \\
& -\frac{1}{4} \delta_{3}\left(F_{r}^{\mu \nu}\right)^{2}+i \delta_{2} \bar{\Psi}_{r} \not \partial \Psi_{r}-\left(\delta_{m}+m \delta_{2}\right) \bar{\Psi}_{r} \Psi_{r}-e \delta_{1} \bar{\Psi}_{r} \gamma_{\mu} \Psi_{r} A_{r}^{\mu},
\end{aligned}
$$

with $z_{3}=1+\delta_{3}, z_{2}=1+\delta_{2}, m_{0}=m+\delta_{m}$ and $z_{1}=1+\delta_{1}$, where $\delta_{1}, \delta_{2}, \delta_{3}$ and $\delta_{m}$ are counterterms. Here, $m$ and $e$ are the physical mass and physical charge of the electron measured at large distances. Now, the Feynman rules for 
the above Lagrangian are:

$$
\underset{\vec{k}}{\sim}=-i e \gamma^{\mu}
$$


We use the following notations:

$$
\rightarrow=-i \Sigma(\not p)
$$

Here '1PI' denotes a one-particle irreducible diagram which is the sum of any diagram that cannot split in two by removing a single line. To fix the pole of the fermion propagator at the physical mass $m$ we need two renormalization conditions (see [14]):

$$
\begin{aligned}
\Sigma(\not p=m) & =0 \\
\left.\frac{d \Sigma(\not p)}{d \not p}\right|_{\not p=m} & =0 .
\end{aligned}
$$

The renormalization condition which fixes the mass of the photon to zero is

$$
\Pi\left(q^{2}=0\right)=0 .
$$

Given the above conditions, finally, the physical electron charge is derived by the following renormalization condition:

$$
-i e \Gamma^{\mu}\left(p^{\prime}-p=0\right)=-i e \gamma^{\mu}
$$

Now, using the dimensional regularization we are able to compute $-i \Sigma(\not p), i \Pi\left(q^{2}\right)$ and $-i e \Gamma^{\mu}\left(p^{\prime}, p\right)$. Applying the above renormalization conditions, up to leading order in $\alpha$, the divergent parts of the counterterms are derived as 43 ]

$$
\begin{aligned}
\delta_{2} & \sim-\frac{e^{2}}{8 \pi^{2} \epsilon}, \\
\delta_{m} & \sim-\frac{3 m e^{2}}{8 \pi^{2} \epsilon}, \\
\delta_{3} & \sim-\frac{e^{2}}{6 \pi^{2} \epsilon}, \\
\delta_{1}=\delta_{2} & \sim-\frac{e^{2}}{8 \pi^{2} \epsilon},
\end{aligned}
$$

where $d=4-\epsilon$ is the spacetime dimension so that we should take the limit $\epsilon \rightarrow 0$. As a matter of fact, these counterterms are able to remove all UV divergences of the QED theory in free space.

\section{RENORMALIZATION IN POSITION SPACE}

In this section we survey the renormalization for QED in coordinate space within the renormalized perturbation theory. Naturally, when a systematic treatment of the renormalization program is done, the counterterms automatically 
turn out to be dependent on the functional form of the fields. In addition, the RG may lead to position dependent mass and charge, as a manifestation of the explicitly broken translational symmetry of the system. It is worth mentioning that our main scheme is in accordance with the standard renormalization approach in momentum space where we have the translational invariance. In the next three subsections we separately consider electron self-energy, photon self-energy and vertex correction, and derive the counterterms by imposing proper renormalization conditions in the configuration space.

\section{Electron Self-Energy}

According to the Lagrangian (6), the perturbation expansion of the full electron propagator up to order $\alpha$ is

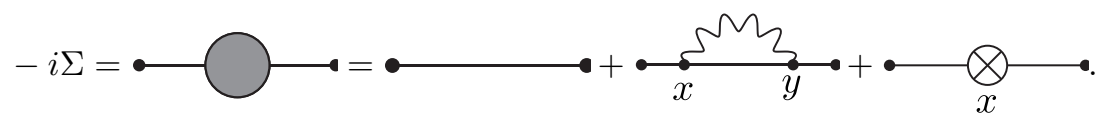

We choose our renormalization condition in such a way that pole of the first term of right hand side (RHS) gives the physical mass $m$ at $x=x_{0}$. This requires that the sum of remaining diagrams, which we call it $-i \tilde{\Sigma}(x)$ vanishes at this point, namely

$$
-\left.i \tilde{\Sigma}(x)\right|_{x=x_{0}}=\left(\bullet \underset{x}{s} \cdot+-\bigotimes_{x} \cdot\right)_{x=x_{0}}=0, \quad \text { and }\left.\quad \frac{d[-i \tilde{\Sigma}(x)]}{d x}\right|_{x=x_{0}}=0
$$

We can write $-i \widetilde{\Sigma}$ to order $\alpha$ as

$$
-i \widetilde{\Sigma}(x)=\int d^{d} y \bar{\psi}(y)\left[-i \Sigma_{2}(x, y)\right] \psi(x)+\bar{\psi}(x)\left[-\delta_{2}(x) \not \partial-i m \delta_{2}(x)-i \delta_{m}(x)\right] \psi(x)
$$

Thus the first condition in Eq. 25] yields

$$
\begin{aligned}
-i \widetilde{\Sigma}\left(x_{0}\right) & =\left\{\int d^{d} y \bar{\psi}(y)\left[-i \Sigma_{2}(x, y)\right] \psi(x)+\bar{\psi}(x)\left[-\delta_{2}(x) \not \partial-i m \delta_{2}(x)-i \delta_{m}(x)\right] \psi(x)\right\}_{x=x_{0}} \\
& =0
\end{aligned}
$$

where $-i \Sigma_{2}$ is $O(\alpha)$ electron self-energy diagram. Now, using Dirac equation $(i \not \partial-m) \psi=0$, up to order $\alpha$ we obtain

$$
\delta_{m}=\left.\frac{-1}{\bar{\psi}\left(x_{0}\right) \psi\left(x_{0}\right)} \int d^{d} y \bar{\psi}(y) \Sigma_{2}(x, y) \psi(x)\right|_{x=x_{0}} .
$$

To simplify the second condition in Eq. 25 we note that the $\widetilde{\Sigma}(x)$ is, in fact, a function of $\bar{\psi}(x), \psi(x), \not \partial \bar{\psi}(x)$ and $\not \partial \psi(x)$ so that

$$
\frac{d \widetilde{\Sigma}(x)}{d x}=\frac{\partial \psi}{\partial x} \frac{\partial \widetilde{\Sigma}}{\partial \psi}+\frac{\partial \bar{\psi}}{\partial x} \frac{\partial \widetilde{\Sigma}}{\partial \bar{\psi}}+\frac{\partial(\not \partial \bar{\psi})}{\partial x} \frac{\partial \widetilde{\Sigma}}{\partial(\not \partial \bar{\psi})}+\frac{\partial(\not \partial \psi)}{\partial x} \frac{\partial \widetilde{\Sigma}}{\partial(\not \partial \psi)}
$$

Due to the opposite sign of the momentum for particles and anti-particles the first two terms in cancel each other. The third term is also zero, because there is no derivative of $\bar{\psi}$ in $\widetilde{\Sigma}$. Thus, we obtain

$$
\left.\frac{\partial[-i \widetilde{\Sigma}(x)]}{\partial(\not \partial \psi)}\right|_{x=x_{0}}=0
$$


We can derive $\delta_{2}\left(x_{0}\right)$ by using the above equation and Eq. 26

$$
\begin{aligned}
\left.\frac{\partial[-i \widetilde{\Sigma}(x)]}{\partial(\not \partial \psi)}\right|_{x=x_{0}} & =\left.\int d^{d} y \frac{\partial\left[\bar{\psi}(y)\left(-i \Sigma_{2}(x, y)\right) \psi(x)\right]}{\partial(\not \partial \psi(x))}\right|_{x=x_{0}}-\bar{\psi}\left(x_{0}\right) \delta_{2}\left(x_{0}\right)=0 \\
\Rightarrow \delta_{2} & =\left.\frac{1}{\bar{\psi}\left(x_{0}\right)} \int d^{d} y \frac{\partial\left[\bar{\psi}(y)\left(-i \Sigma_{2}(x, y)\right) \psi(x)\right]}{\partial(\not \partial \psi(x))}\right|_{x=x_{0}} .
\end{aligned}
$$

\section{Photon Self-Energy}

For the photon propagator we again expand the full propagator as

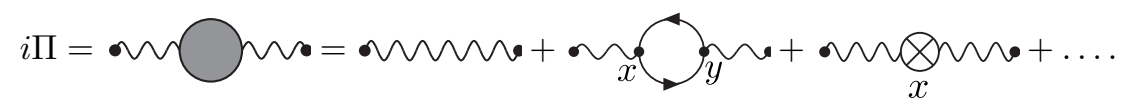

To have a massless photon, at $x=x_{0}$, we need only the first term on the RHS with a pole which definitely fixed on zero. Therefore, the rest of the perturbation series must vanish, so that up to order $\alpha$ we have

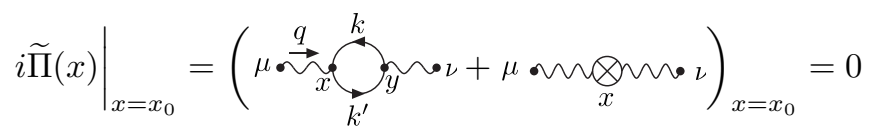

or equivalently,

$$
i \widetilde{\Pi}\left(x_{0}\right)=\left\{\int d^{d} y \widetilde{A}_{\mu}^{*}(y)\left[i \Pi_{2}^{\mu \nu}(x, y)\right] \widetilde{A}_{\nu}(x)+\widetilde{A}_{\mu}^{*}(x) \delta_{3}(x)\left[-i\left(g^{\mu \nu} \partial^{2}-\partial^{\mu} \partial^{\nu}\right)\right] \widetilde{A}_{\nu}(x)\right\}_{x=x_{0}}=0,
$$

where $i \Pi_{2}^{\mu \nu}(x, y)$ is $O(\alpha)$ photon self-energy diagram. Therefore,

$$
\begin{gathered}
\delta_{3}=\left.\int d^{d} y \frac{-\widetilde{A}_{\mu}^{*}(y) \Pi_{2}^{\mu \nu}(x, y) \widetilde{A}_{\nu}(x)}{\widetilde{A}_{\mu}^{*}(x)\left(g^{\mu \nu} \partial^{2}-\partial^{\mu} \partial^{\nu}\right) \widetilde{A}_{\nu}(x)}\right|_{x=x_{0}} . \\
\text { 3. Vertex Correction }
\end{gathered}
$$

Formally, the vertex corrections give us the physical charge of electron. Diagrammatically we have

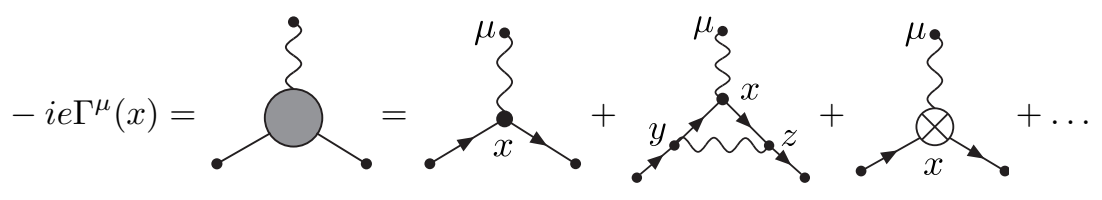

Our renormalization condition for the electron charge is to fix it on physical $e$ at $x=x_{0}$. We can do this by using the first term on RHS of Eq. (38), so that the remaining diagrams should cancel each other, 


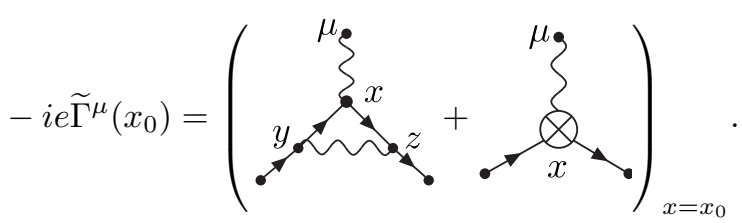

We can equivalently write the above equation as,

$$
-i e \widetilde{\Gamma}^{\mu}\left(x_{0}\right)=\left\{\int d^{d} y d^{d} z \bar{\psi}(z)\left[-i e \delta \Gamma^{\mu}(x, y, z)\right] \psi(y)+\bar{\psi}(x)\left[-i e \delta_{1}(x) \gamma^{\mu}\right] \psi(x)\right\}_{x=x_{0}}=0
$$

where $-i e \delta \Gamma^{\rho}$ is the vertex correction diagram to order $\alpha$. Therefore we find

$$
\delta_{1} \gamma^{\mu}=\left.\int d^{d} y d^{d} z \frac{\bar{\psi}(z) \delta \Gamma^{\mu}(x, y, z) \psi(y)}{\bar{\psi}(x) \psi(x)}\right|_{x=x_{0}}
$$

Accordingly we may derive counterterms required for renormalzation of QED in coordinate space. These counterterms could be applied for problems in which the translational invariance breaks explicitly. Obviously if we work in free space, with the translational symmetry, they should reduce to those in the standard prevalent derived in momentum space. We show this equivalence in the next section.

\section{COMPARISON TO MOMENTUM SPACE (FREE SPACE)}

In this section, as a special case, we compare our results with renormalization of QED in free space. In free space, the wave functions of fermions and photons are considered as plane waves. We start with the Eq. (29) by inserting $\psi(x)=u^{s}(p) e^{-i p \cdot x}$ (from here on we drop the superscript $s$ for simplicity). Then, the numerator of the integrand becomes

$$
\begin{aligned}
\int d^{d} y \bar{\psi}(y)\left[-i \Sigma_{2}(x, y)\right] \psi(x) & =-e^{2} \int d^{d} y \bar{u}(p) e^{i p \cdot y} \gamma^{\mu} S(x-y) \gamma^{\nu} D_{\mu \nu}(y-x) u(p) e^{-i p \cdot x} \\
& =-e^{2} \bar{u}(p)\left[\int d^{d} y \frac{d^{d} k}{(2 \pi)^{d}} \frac{d^{d} k^{\prime}}{(2 \pi)^{d}} \gamma^{\mu} \frac{\not k-m}{k^{2}-m^{2}} \frac{\gamma_{\mu}}{k^{\prime 2}} e^{-i\left(k+k^{\prime}-p\right) \cdot y} e^{\left.-i\left(p-k^{\prime}-k\right) \cdot x\right] u(p),(}\right]
\end{aligned}
$$

where $S(x-y)$ and $D^{\mu \nu}(y-x)$ are the propagators of fermion and photon in $d$ spacetime dimensions, respectively,

$$
S(x-y)=\int \frac{d^{d} k}{(2 \pi)^{d}} \frac{i}{\not k-m} e^{-i k \cdot(x-y)},
$$

and,

$$
D^{\mu \nu}(x-y)=\int \frac{d^{d} k}{(2 \pi)^{d}} \frac{-i g^{\mu \nu}}{k^{2}} e^{-i k \cdot(x-y)} .
$$

Integrating over position and then $k^{\prime}$ in Eq. 42 yields

$$
\int d^{d} y \bar{\psi}(y)\left[-i \Sigma_{2}(x, y)\right] \psi(x)=-e^{2} \bar{u}(p)\left[\int \frac{d^{d} k}{(2 \pi)^{d}} \gamma^{\mu} \frac{1}{\not k-m} \gamma_{\mu} \frac{1}{(p-k)^{2}}\right] u(p) .
$$

In terms of $\epsilon=4-d$, the above equation becomes

$$
\begin{aligned}
\int d^{d} y \bar{\psi}(y)\left[-i \Sigma_{2}(x, y)\right] \psi(x) & =\bar{u}(p) \frac{-i e^{2}}{8 \pi^{2} \epsilon}(-\not p+4 m) u(p)+O\left(\epsilon^{0}\right) \\
& =\frac{-3 i m e^{2}}{8 \pi^{2} \epsilon} \bar{u}(p) u(p)+O\left(\epsilon^{0}\right) .
\end{aligned}
$$


Finally, using Eq. (29) we have

$$
\begin{aligned}
\delta_{m} & =\frac{-1}{\bar{u} u} \frac{3 m e^{2} \bar{u} u}{8 \pi^{2} \epsilon}+O\left(\epsilon^{0}\right) \\
& =\frac{-3 m e^{2}}{8 \pi^{2} \epsilon}+O\left(\epsilon^{0}\right),
\end{aligned}
$$

The above result is clearly independent of $x_{0}$, manifesting the translational invariance of the system. It is also in agreement with Eq. (21), the standard common counterterm derived directly in free space.

We similarly derive the second counterterm, $\delta_{2}$. Now, using Eq. 46 and the fact that $\not \partial \psi=\not \partial\left[u(p) e^{-i p . x}\right]=-i \not p \psi$, we can rewrite the Eq. (33) as follows:

$$
\delta_{2}=\frac{1}{\bar{u}(p)} \frac{\partial\left[\bar{u}(p) \frac{-i e^{2}}{8 \pi^{2} \epsilon}(-\not p+4 m) u(p)\right]}{\partial[-i \not p u(p)]}=\frac{-e^{2}}{8 \pi^{2} \epsilon}+O\left(\epsilon^{0}\right),
$$

which is precisely in agreement with Eq. 20]. Again we see that the position dependence cancels out as expected.

To compute $\delta_{3}$ in free space, we use $\widetilde{A}_{\mu}(p, x)=\varepsilon_{\mu}^{s}(p) e^{-i p . x}$ in Eq. (37). The numerator becomes

$$
\begin{aligned}
\int d^{d} y \widetilde{A}_{\mu}^{*}(y)\left(i \Pi_{2}^{\mu \nu}\right) \widetilde{A}_{\nu}(x) & =\varepsilon_{\mu}^{*}\left[-i e^{2} \int d^{d} y \gamma^{\mu} S(x-y) \gamma^{\nu} S(y-x) e^{-i q \cdot x} e^{i q \cdot y}\right] \varepsilon_{\nu} \\
& =\varepsilon_{\mu}^{*}\left[i e^{2} \int d^{d} y \int \frac{d^{d} k}{(2 \pi)^{d}} \frac{d^{d} k^{\prime}}{(2 \pi)^{d}} \gamma^{\mu} \frac{1}{\not k-m} \gamma^{\nu} \frac{1}{\not k^{\prime}-m} e^{-i\left(q+k-k^{\prime}\right) \cdot x} e^{\left.-i\left(-k-q+k^{\prime}\right) \cdot y\right] \varepsilon_{\nu}}\right.
\end{aligned}
$$

Integrating over $y$ and $k^{\prime}$, the RHS gives,

$$
\varepsilon_{\mu}^{*}\left[i e^{2} \int \frac{d^{d} k}{(2 \pi)^{4}} \gamma^{\mu} \frac{1}{\not k-m} \gamma^{\nu} \frac{1}{\not q+\not k-m}\right] \varepsilon_{\nu} .
$$

By simple calculations we finally have,

$$
\int d^{d} y \widetilde{A}_{\mu}^{*}\left(i \Pi_{2}^{\mu \nu}\right) \widetilde{A}_{\nu}=\varepsilon_{\mu}^{*} \frac{-i e^{2}}{6 \pi^{2} \epsilon}\left(g^{\mu \nu} k^{2}-k^{\mu} k^{\nu}\right) \varepsilon_{\nu}+O\left(\epsilon^{0}\right) .
$$

Inserting the above calculation in Eq. (37) and using $\widetilde{A}_{\mu}^{*}(x) \widetilde{A}_{\nu}(x)=\varepsilon_{\mu}^{*} \varepsilon_{\nu}$ we derive,

$$
\begin{aligned}
\delta_{3} & =\frac{-i e^{2}}{6 \pi^{2} \epsilon} \frac{\varepsilon_{\mu}^{*}\left(g^{\mu \nu} k^{2}-k^{\mu} k^{\nu}\right) \varepsilon_{\nu}}{\widetilde{A}_{\mu}^{*}\left[-i\left(g^{\mu \nu}\left(-k^{2}\right)+k^{\mu} k^{\nu}\right)\right] \widetilde{A}_{\nu}}+O\left(\epsilon^{0}\right) \\
& =-\frac{e^{2}}{6 \pi^{2} \epsilon}+O\left(\epsilon^{0}\right),
\end{aligned}
$$

which is in accordance with Eq. (22).

For the last counterterm, $\delta_{1}$, the numerator in Eq. (41) can be rewritten as

$$
\begin{gathered}
\int d^{d} z d^{d} y \bar{\psi}(z) \delta \Gamma^{\mu}(x, y, z) \psi(y)=-e^{2} \int d^{d} z d^{d} y \bar{\psi}\left(p^{\prime}, z\right) \gamma^{\alpha} S(z, x) \gamma^{\mu} S(x, y) \gamma^{\beta} \psi(p, y) D_{\alpha \beta}(y, z) \\
=e^{2} \int d^{d} z d^{d} y \bar{u}\left(p^{\prime}\right)\left[e^{i p^{\prime} \cdot z} \int \frac{d^{d} k}{(2 \pi)^{d}} \frac{d^{d} k^{\prime}}{(2 \pi)^{d}} \frac{d^{d} k^{\prime \prime}}{(2 \pi)^{d}}\right. \\
\left.\times \gamma^{\alpha} \frac{e^{-i k^{\prime} \cdot(z-x)}}{\not k^{\prime}-m} \gamma^{\mu} \frac{e^{-i k \cdot(x-y)}}{\not k-m} \gamma^{\beta} e^{-i p \cdot y} \frac{-i g_{\alpha \beta}}{k^{\prime \prime 2}} e^{\left.-i k^{\prime \prime} .(z-y)\right] u(p)}\right] \\
=-i e^{2} \bar{u}\left(p^{\prime}\right) \int \frac{d^{d} k}{(2 \pi)^{d}} \frac{d^{d} k^{\prime}}{(2 \pi)^{d}} \frac{d^{d} k^{\prime \prime}}{(2 \pi)^{d}} \gamma^{\alpha} \frac{1}{\not k^{\prime \prime}-m} \gamma^{\mu} \frac{1}{\not k-m} \gamma^{\beta} \frac{g_{\alpha \beta}}{k^{\prime \prime 2}} \\
\times(2 \pi)^{2 d} \delta^{(d)}\left(k+k^{\prime \prime}-p\right) \delta^{(d)}\left(p^{\prime}-k^{\prime}-k^{\prime \prime}\right) e^{i\left(k^{\prime}-k\right) \cdot x} u(p) .
\end{gathered}
$$


Taking integral of $k^{\prime}$ and $k^{\prime \prime}$ yields,

$$
\begin{aligned}
\int d^{d} z d^{d} y \bar{\psi}(z) \delta \Gamma^{\mu}(x, y, z) \psi(y) & =-i e^{2} \bar{u}\left(p^{\prime}\right)\left[\int \frac{d^{d} k}{(2 \pi)^{d}} \gamma^{\alpha} \frac{1}{\not p^{\prime}-m} \gamma^{\mu} \frac{1}{\not k-m} \gamma^{\beta} \frac{-g_{\alpha \beta}}{(p-k)^{2}} e^{i\left(p^{\prime}-p\right) \cdot x}\right] u(p) \\
& =\bar{u}\left(p^{\prime}\right)\left[\frac{-e^{2}}{8 \pi^{2} \epsilon} \gamma^{\mu} e^{i\left(p^{\prime}-p\right) \cdot x}\right] u(p)+O\left(\epsilon^{0}\right) .
\end{aligned}
$$

Replacing this result in Eq. (41) we find,

$$
\begin{aligned}
\delta_{1} \gamma^{\mu} & =\left.\int d^{d} z d^{d} y \frac{\bar{\psi}(y) \delta \Gamma^{\mu}(x, y, z) \psi(x)}{\bar{\psi}(x) \psi(x)}\right|_{x=x_{0}}=\frac{\bar{u}\left(p^{\prime}\right)\left[\frac{-e^{2}}{8 \pi^{2} \epsilon} \gamma^{\mu} e^{i\left(p^{\prime}-p\right) \cdot x_{0}}\right] u(p)}{\bar{u}\left(p^{\prime}\right) u(p) e^{i\left(p^{\prime}-p\right) \cdot x_{0}}}+O\left(\epsilon^{0}\right) \\
& \Rightarrow \delta_{1}=-\frac{e^{2}}{8 \pi^{2} \epsilon}+O\left(\epsilon^{0}\right),
\end{aligned}
$$

which is again in complete agreement with Eq. (23). This counterterm is equal to $\delta_{2}$ as it should be, due to the Ward identity. Consequently, up to order $\alpha$, we show that our counterterms in position space are equal to the usual terms derived in momentum space. Obviously, the results, in this case, do not depend on the special point $x_{0}$ where our renormalization conditions are imposed, manifesting the translational invariance of this problem.

\section{CONCLUSIONS}

Ultraviolet infinities of QED theory are basically due to three divergent Feynman diagrams: vertex correction, vacuum polarization and electron self-energy. Using renormalization program, in free space with translational symmetry, these infinities are controlled by four counterterms which are generally derived in momentum space. However, if the translational invariance of the system is broken strongly then the momentum is no longer a good quantum number. Renormalization procedure in configuration space can be applied for such a situation, for example, in problems with a nontrivial $\mathrm{BC}$ or a nonzero background which cannot be treated as small perturbations. In this paper, we have done the renormalization in real space in the presence of nontrivial BC and derived the form of four counterterms up to order $\alpha$. Systematic treatment of the renormalized perturbation theory after imposing renormalization conditions leads us to $x$-independent counterterms which directly indicate the dependency on the BCs of the fermion and photon fields. Finally, as a particular case, our results have been compared with those obtained in free space and we have shown the equivalence in the two cases is guaranteed, up to order $\alpha$.

[1] J. Schwinger, Electron Anomaly to Order $\alpha$, Phys. Rev. 73 (1948) 416.

[2] H. A. Kramers, Non-relativistic Quantum-electrodynamics and Correspondence Principle, Proceedings Solvay Conference "Les Particules Elmentaires" (1948).

[3] Tomonaga, S. "On a Relativistically Invariant Formulation of the Quantum Theory of Wave Fields." Prog. Theor. Phys. 1 (1946) 27.

[4] Luis Alvarez-Gaume, Miguel A. Vazqez-Mozo, An Invitation to Quantum Field Theory, Springer (2010).

[5] Ling-Fong Li, Chongqing, Introduction to Renomalization in Field Theory, arXiv:1208.4700v1 (2012).

[6] M. Böhm, H. Spiesberger, W. Hollik, On the 1Loop Renormalization of the Electroweak Standard Model and its Application to Leptonic Processes, Fortschritte der Physik, 34 (1986) 687.

[7] G. Martinelli, C. Pittori, C. T. Sachrajda, M. Testa, A. Vladikas, A General Method for Non-perturbative Renormalization of Lattice Operators, Nucl. Phys B443 (1995) 81.

[8] F. Mandl, G. Shaw: Quantum Field Theory, Revised Edition, John Wiley and Sons (1993).

[9] F. Halzen, A. D. Martin, Quarks and Leptons: An Introductory Course in Modern Particle Physics, John Wiley and Sons Publication (1984).

[10] R. Stora, Renormalized Perturbation Theory: a Missing Chapter, Int. J. Geom. Methods Mod. Phys. 05 (2008) 1345.

[11] O. Piguet, S. P. Sorella, Algebraic Renormalization, Springer Publication (1995).

[12] M. S. Schwarts, Quantum Field Theory and the Standard Model, Cambridge University Press (2014).

[13] G. B. Folland, Quantum Field Theory: A Tourist Guide for mathematicians, American Mathematical Society (2008).

[14] M. D. Schwartz, Quantum Field Theory and the Standard Model, Cambridge University Press (2014).

[15] I. J. R. Aitchison, A. J. G. Hey: Gauge Theories in Particle Physics, A Practical Introduction, Third Edition. Volume I: From Relativistic Quantum Mechanics to QED, Taylor and Francis Group, LLC (2003).

[16] A. Zee, Quantum Field Theory in a Nutshell, Princeton University Press (2010). 
[17] F. J. Dyson, The Radiation Theories of Tomonaga, Schwinger, and Feynman, Phys. Rev. 75 (1949) 486.

[18] J. Schwinger, Selected Papers on Quantum Electrodynamics, New York, Dover Publication (1958).

[19] C. Brouder, Renormalization of QED in an External Field, EPJ Direct 4 (2002) 1.

[20] T. Lancaster, S. J. Blundell, Quantum Field Theory for the Gifted Amateur, Oxford University Press (2014).

[21] J. Collins, Foundations of Perturbative QCD, Cambridge University Press (2011).

[22] Gerardt Hooft, Gauge Theory and Renormalization, History of Original Ideas and Basic Discoveries in Particle Physics, 352 (1996) 37.

[23] T. Luthe, A. Maier, P. Marquar, Y. Schröder, Complete Renormalization of QCD at Five Loops, JHEP 03 (2017) 020.

[24] S. Coleman, Quantum Sine-Gordon Equation as the Massive Thirring Model, Phys. Rev. D 11 (1975) 2088.

[25] R. F. Dashen, B. Hasslacher, and A. Neveu, Nonperturbative Methods and Extended-hadron Models in Field Theory. II. Two-dimensional Models and Extended Hadrons, Phys. Rev. D 10 (1974) 4130.

[26] E. Brèzin, J. C. Le Guillou, and J. Zinn-Justin, Perturbation Theory at Large Order. I. The $\phi^{2 N}$ Interaction, Phys. Rev. D 15 (1977) 1544.

[27] E. Brèzin, G. Parisi, Critical Exponents and Large-order Behavior of Perturbation Theory, J. Stat. Phys. 19 (1978) 269.

[28] S. Weinberg, The Quantum Theory of Fields, Volume 2: Modern Applications, Cambridge University Press (2010).

[29] T. Y. Cao, Conceptual Foundations of Quantum Field Theory, Cambridge University Press (2004).

[30] T. J. Hollowood, Renormalization Group and Fixed Points: in Quantum Field Theory, Springer Publisher (2013).

[31] S. Coleman, Aspects of Symmetry, Cambridge University Press (1985).

[32] O. J. Rosten, Fundamentals of the Exact Renormalization Group, Phys. Rep. 511 (2012) 177.

[33] R. Moazzemi, S. S. Gousheh, A Renormalized Perturbation Theory for Problems with Nontrivial Boundary Conditions or Backgrounds in Two Space-time Dimensions, Eur. Phys. J. C 56 (2008) 585.

[34] D. Z.Freedman, K. Johnson and J. Latorre, "Differential regularization and renormalization: A New method of calculation in quantum field theory", Nucl. Phys. B, 371 (1992) 353.

[35] D. Z. Freedman, K. Johnson, R. Munoz-Tapia, and X. Vilasis-Cardona, "A Cutoff procedure and counterterms for differential renormalization" Nucl. Phys. B 395 (1993) 454.

[36] F. del Aguila, M. Perez-Victor, "Constrained differential renormalization, Acta Phys. Polon. B28 (1997) 2279.

[37] V.A. Smirnov, "Renormalization without regularization" Theor. Math. Phys. 117 (1998) 1368.

[38] C. R. Pontes, A. B. Scarpelli, M. Sampaio, J. L. Acebal and M. C. Nemes, "On the equivalence between implicit regularization and constrained differential renormalization", The Euro. Phys. J. C 53 (2008) 121.

[39] V. A. Smirnov, "Differential renormalization and dimensional regularization", Nucl. Phys. B 427 (1994) 325.

[40] G. Dunne and N. Rius, "A Comment on the relationship between differential and dimensional renormalization", Phys. Lett. B 293 (1992) 367.

[41] J. C. Collins, Renormalization, Cambridge University of Press (1984).

[42] Michael E. Peskin, Daniel V. Schroeder: An Introduction to Quantum Field Theory, Westview Press (1995).

[43] L. H. Ryder, Quantum Field Theory, Second Edition, Cambridge University Press (1996).

[44] C. Itzykson, J. B. Zuber, Quantum Field Theory, McGraw-Hill Publisher (1980).

[45] M. Bohm, A. Denner, H. Joos, Gauge Theories of the Strong and Electroweak Interaction, Springer Publisher (2001) .

[46] P. E. Haagensen and J. I. Latorre, "Differential renormalization of massive quantum field theories", Phys. Lett. B 283 (1992) 293.

[47] P. E. Haagensen and J. I. Latorre, "A Comprehensive Coordinate Space Renormalization of Quantum Electrodynamics toto Two-Loop Order", Ann. Phys. 221 (1993) 77.

[48] E. M. Malatesta, G. Parisi, T. Rizzo, Two-loop Corrections to Large Order Behavior of $\phi^{4}$ Theory, Nucl. Phys B922 (2017) 293.

[49] R. Moazzemi, and S. S. Gousheh, A New Renormalization Approach to the Dirichlet Casimir Effect for $\phi^{4}$ Theory in $(1+1)$ Dimensions, Phys. Lett. B658 (2008) 255.

[50] S. S. Gousheh, R. Moazzemi, M. A. Valuyan, Radiative Correction to the Dirichlet Casimir Energy for $\lambda \phi^{4}$ Theory in Two Spatial Dimensions, Phys. Lett. B681 (2009) 477.

[51] R. Moazzemi, M. Namdar, S. S. Gousheh, The Dirichlet Casimir Effect for $\phi^{4}$ Theory in (3+1) Dimensions: A New Renormalization Approach, JHEP 09, (2007) 029.

[52] S.S. Gousheh, A. Mohammadi, M. Asghari, R. Moazzemi, F. Charmchi, The Radiative Corrections to the Mass of the Kink Using an Alternative Renormalization Program, JHEP 07 (2012) 060.

[53] R. M. Wald, Quantum Field Theory in Curved Spacetime and Black Hole Thermodynamics, the University of Chicago Press (1994).

[54] K. Frednhagen and K. Rejzner, QFT on Curved Spacetimes: Axiomatic Framework and Examples, J. Math. Phys. 57 (2016) 031101.

[55] I. Todorov, Renormalization of Position Space Amplitudes in a Massless QFT, Physics of Particles and Nuclei 48 (2017) 227.

[56] I. Todorov, Relativistic Causality and Position Space Renormalization, Nuclear Physics B912 (2016) 79. 